\title{
DNA sequence features that may establish H3K27ac mark
}

\author{
A. Zubritskiy ${ }^{1 *}$, Y.A. Medvedeva ${ }^{1,2,3}$ \\ ${ }^{1}$ Institute of Bioengineering, Research Centre of Biotechnology, RAS, Moscow, Russia \\ ${ }^{2}$ Department of System Biology and Bioinformatics, Vavilov institute of General Genetics, RAS, \\ Moscow, Russia \\ ${ }^{3}$ Department of Biological and Medical Physics, Moscow Institute of Physics and Technology, \\ Dolgoprudny, Russia \\ *e-mail:a.zubrit@gmail.com
}

Key words: histone modification, $\mathrm{H} 3 \mathrm{~K} 27 \mathrm{ac}, \mathrm{CpG}$ dinucleotide

Motivation and Aim: Histone posttranslation modification (PTM) is a key mechanism of epigenetic regulation. Histone PTMs are highly variable, but in some genomic loci they may be highly similar between many cell lines. It has been reported that the attraction of polycomb repressive complex 2 (PRC2) and consequent trimethylation of Lys27 of histone $\mathrm{H} 3$ (H3K27me3) is correlated with the local density of $\mathrm{CpG}$ dinucleotides [1]. Other sequence patterns, such as transcription factor (TF) binding sites (TFBS), also affect the presence of histone modifications: the ENCODE project demonstrated a specific histone modification profile around binding sites of many TFs [2].

Another histone PTM, acetylated Lys27 of histone H3 (H3K27ac) is mutually exclusive with $\mathrm{H} 3 \mathrm{~K} 27 \mathrm{me} 3$, but it is still unclear if $\mathrm{H} 3 \mathrm{~K} 27 \mathrm{ac}$ shares any sequence specific pattern. In this work we performed a direct experiment to test whether a specific genomic sequence is capable of recovering $\mathrm{H} 3 \mathrm{~K} 27 \mathrm{ac}$.

Methods and Algorithms: We generated stably transfected Cakil cell line that contain selection cassette flanked by LoxP sites in the beta-globin locus. This cassette can be exchanged via Cre-mediated recombination to any other DNA sequence of interest flanked by LoxP sites.

Results: We performed insertion of 10 DNA sequences into beta-globin locus. This step could help us to unify the epigenetic background and remove any preceding histone PTMs from DNA fragments. 3 of inserted sequences are GC-rich promotor regions with high number of $\mathrm{CpG}$ dinucleotides, while other DNA sequences have less than two CpG's per fragment and CG content in range between 27 and $68 \%$.

Surprisingly, none of the GC- and $\mathrm{CpG}$ rich promoter regions, that were acetylated in their original genomic loci recovered $\mathrm{H} 3 \mathrm{~K} 27 \mathrm{ac}$ after relocation to a beta globin locus, while two extremely GC-rich but $\mathrm{CpG}$ poor sequences gained $\mathrm{H} 3 \mathrm{~K} 27 \mathrm{ac}$ in the beta globin locus, while in their native location they had no H3K27ac.

Conclusion: The establishment of H3K27ac may not depend on a CpG content of the sequence per se, as opposed to its antagonistic mark H3K27me3, but might depend on an elevated GC content.

Acknowledgements: Supported by the grant from Russian Science Foundation (15-1430002).

\section{References}

1. Jermann P. et al. (2014) Short sequences can efficiently recruit histone H3 lysine 27 trimethylation in the absence of enhancer activity and DNA methylation. Proc Natl Acad Sci USA. 111(33):E3415-3421.

2. Hoffman M.M. et al. (2013) Integrative annotation of chromatin elements from ENCODE data. Nucleic Acids Res. 41(2):827-841. 